\title{
Longer operant lever-press duration requirements induce fewer but longer response bouts in rats
}

\author{
Ryan J. Brackney ${ }^{1,2} \cdot$ Raul Garcia $^{1,3} \cdot$ Federico Sanabria ${ }^{4}$ \\ Accepted: 14 January 2021 / Published online: 24 February 2021 \\ (C) The Psychonomic Society, Inc. 2021
}

\begin{abstract}
Operant behavior is organized in bouts that are particularly visible under variable-interval (VI) schedules of reinforcement. Previous research showed that increasing the work required to produce a response decreases the rate at which bouts are emitted and increases the minimum interresponse time (IRT). In the current study, the minimum effective IRT was directly manipulated by changing the minimum duration of effective lever presses reinforced on a VI 40-s schedule. Contrary to assumptions of previous models, response durations were variable. Response durations were typically $0.5 \mathrm{~s}$ greater than the minimum duration threshold; durations that exceeded this threshold were approximately log-normally distributed. As the required duration threshold increased, rats emitted fewer but longer bouts. This effect may reflect an effort-induced reduction in motivation and a durationinduced facilitation of a response-outcome association.
\end{abstract}

Keywords Bouts $\cdot$ Duration $\cdot$ Refractory period $\cdot$ Variable interval $\cdot$ Lever press $\cdot$ Rats

Operant behavior is organized in response bouts (e.g., Daniels \& Sanabria, 2017a; Hoffman \& Newland, 2016; Íbias, Daniels, Miguéns, Pellón, \& Sanabria, 2017; Matsui, Yamada, Sakagami, \& Tanno, 2018; Romero, Daniels, Gipson, \& Sanabria, 2018; Smith, McLean, Shull, Hughes, \& Pitts, 2014). Such organization implies that operant behavior can be described using three parameters: the rate at which bouts are initiated $(b)$, the rate at which responses are emitted within bouts $(w)$, and the mean length of a bout $(L)$. To understand how operant behavior adapts to environmental demands, it is important to establish the differential sensitivity of these parameters to various classes of manipulations (Sanabria, Daniels, Gupta, \& Santos, 2019). Research so far suggests that $b$ is uniquely sensitive to changes in reinforcer efficacy (Brackney, Cheung, Neisewander, \& Sanabria, 2011; Brackney, Cheung, \& Sanabria, 2017; Daniels \& Sanabria,

Federico Sanabria

Federico.Sanabria@asu.edu

1 Interdisciplinary Graduate Degree Program in Neuroscience, Arizona State University, Tempe, AZ, USA

2 Present address: Square, Inc., San Francisco, CA, USA

3 Present address: Neuronal Networks Section, National Institute on Drug Abuse, Bethesda, MD, USA

4 Department of Psychology, Arizona State University, P.O. Box 871104, Tempe, AZ 85287-1104, USA 2017b; Johnson, Pesek, \& Christopher Newland, 2009; Shull, 2004; Shull, Gaynor, \& Grimes, 2001) and rate of reinforcement (Brackney et al., 2017; Cheung, Neisewander, \& Sanabria, 2012; Reed, 2011, 2015; Reed, Smale, Owens, \& Freegard, 2018; Shull et al., 2001; Shull \& Grimes, 2003; Shull, Grimes, \& Bennett, 2004), and $w$ and $L$ are sensitive to changes in contingency requirements (Brackney et al., 2011; Brackney et al., 2017; Brackney \& Sanabria, 2015; Chen \& Reed, 2020; Reed, 2011; Reed et al., 2018; Shull et al., 2001; Shull \& Grimes, 2003; Shull et al., 2004; Tanno, 2016). Thus, the parameters of the microstructure of operant behavior appear to index two of the three necessary conditions for operant performance (Killeen, 1994; Killeen \& Sitomer, 2003; Sanabria, 2019): incentive motivation (b) and response-outcome association learning ( $w$ and $L$ ). The third necessary condition is sensorimotor capacity - the sensory capability to detect stimuli and the motor capability to emit responses that are relevant to the operant task. The present study aims at establishing whether another parameter in the microstructure of operant behavior may index sensorimotor capacity.

The mathematical principles of reinforcement (MPR; Killeen, 1994; Killeen \& Sitomer, 2003) suggest that changes in sensorimotor capacity affect operant performance by setting a ceiling to response rate (Killeen, Hall, Reilly, \& Kettle, 2002), a notion akin to the asymptotic response rate in Herrnstein's (1970) hyperbola. The reciprocal of that ceiling 
is the shortest interresponse time (IRT) possible, which may represent a refractory period $\delta$ during which the subject completes one response and prepares for the next response (see Fig. 1a-b). It has been suggested that every IRT contains a fixed period $\delta$ that is selectively sensitive to changes in motoric requirements (Brackney et al., 2011; Cheung et al., 2012). According to this hypothesis, $w$ is more sensitive to motoric requirements than $b$ (e.g., Jiménez, Sanabria, \& Cabrera, 2017; Johnson et al., 2009) only because $\delta$ constitutes a larger portion of within-bout IRTs than of betweenbout IRTs.

In support of the motoric hypothesis of $\delta$, Brackney et al. (2011) found that increasing the lever height and force requirement for rats responding on a schedule of reinforcement increases $\delta$. However, they also found that this lever manipulation decreases the rate of bout initiations, $b$. Similarly, Posadas-Sanchez (2004) found that increasing the lever force requirement increased MPR-based estimates of $\delta$, but also reduced MPR-based estimates of incentive motivation. Although they did not track changes in $\delta$ with lever height, Jiménez et al. (2017) found that, when response duration is subtracted from IRTs, not only $b$ but also $w$ and $L$ decline when the lever is sufficiently high. Pinkston and Moore (2020) estimated the effect of force requirement on these parameters in rats responding for food, based also on the distribution of IRTs without response durations. They found that raising the force requirement selectively reduced $b$, but only if subcriterial responses (recorded responses with peak force below requirement) were excluded. This effect on $b$ vanished when subcriterial responses were included in the analysis, suggesting that the effects of motoric demands on the parameters of behavioral microstructure are artifactual. It is thus unclear what role motoric demands such as lever height and force requirement play in the microstructure of operant behavior.
A possible explanation for the effect of motoric manipulations on the microstructural parameters of operant behavior suggests an indirect link between these variables: Perhaps the shortest IRTs are sensitive only to changes in motoric requirements (as the motoric hypothesis of $\delta$ postulates), but changes in the shortest IRTs impact the other microstructural parameters. To test this possibility, the current study directly manipulated the required response duration (and, through it, the effective $\delta$ ) to determine its effect on the microstructural parameters of operant behavior. Rats were trained to respond on a variable interval (VI) 40-s schedule of reinforcement. Responses that met or exceeded a duration threshold of 0.0 , 0.4 , or $0.8 \mathrm{~s}$ (depending on the condition) were signaled by a brief tone and light flash. Only signaled responses could trigger reinforcement after the end of the variable interval.

A secondary goal of this study was to characterize the distribution of response durations emitted under a VI schedule. Whereas the motoric hypothesis of $\delta$ assumes that the time to emit a response is fixed, direct measurements of lever-press durations have found them to vary between responses (Byrne \& Sarno, 2019; Fowler, Filewich, \& Leberer, 1977; Roberts \& Gharib, 2006; see Fig. 1c). This study examines the distribution of response durations across different duration thresholds and discusses its implications for response-bout modeling.

\section{Method}

\section{Subjects}

Eight male Wistar rats (WI/NCrl; Charles River Laboratories, USA), starting at postnatal day age 74, partook in the study. All rats were pair-housed and had ad libitum access to food and water. Rats were housed on a reverse dark-light cycle

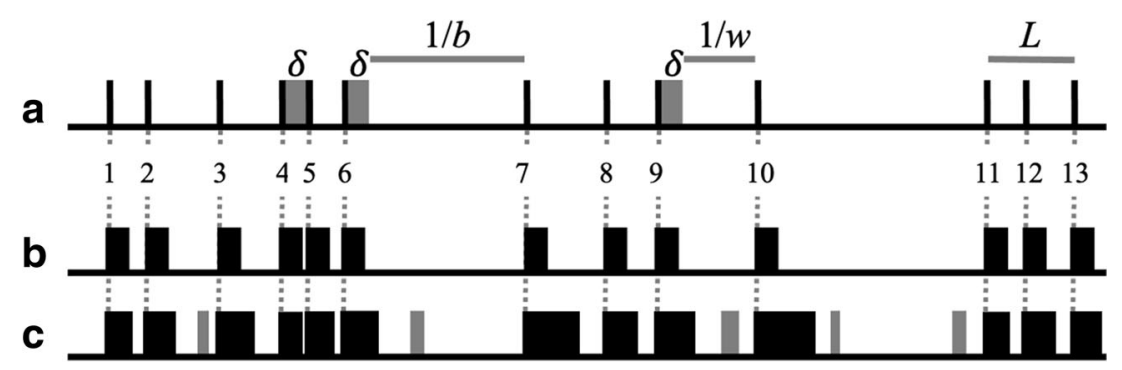

Fig. 1 Illustrative timeline of operant behavior and parameters of its microstructure. a Responses are typically recorded as discrete events of negligible duration (black vertical bars). A refractory period between consecutive responses, $\delta$, is estimated as the shortest interresponse time (IRT) observed; in this illustration, the shortest IRT separates Responses 4 and 5. For the estimation of other parameters, $\delta$ is subtracted from every IRT (gray blocks after Responses 6 and 8 ). The bout initiation rate, $b$, is estimated as the mean reciprocal of IRTs (without $\delta$ ) separating consecutive bouts (e.g., Responses 6 and 7). The within-bout response rate, $w$, is estimated as the reciprocal of IRTs (without $\delta$ ) within bouts (e.g., Responses 9 and 10). Bout length is estimated as the mean number of responses in a bout (e.g., Responses 11, 12, and 13). b Parameter estimation assumes a fixed $\delta$. Because $\delta$ includes the response duration, parameter estimation assumes responses of fixed duration (black blocks). c It is possible, however, that response durations, and thus refractory periods, vary between responses. If a duration threshold is imposed, subthreshold responses (gray blocks) would not be recorded 
(lights off 7 a.m. to 7 p.m.); experiments were conducted during the dark phase of this cycle. Rats had previously been trained to respond on left and right levers in an operant chamber on a VI 120-s schedule of food reinforcement. The study adhered to Arizona State University Institutional Animal Care and Use Committee guidelines.

\section{Apparatus}

Experimental sessions took place in eight Med Associates modular test chambers using the standard operant setup. The chambers were enclosed in a light and sound attenuating box with interior dimensions of $30.5 \mathrm{~cm} \times 24.1 \mathrm{~cm} \times 21.0 \mathrm{~cm}$. All test chambers were controlled by MED-PC® IV software (Med Associates, St. Albans, VT). The operant chambers consisted of a clear polycarbonate roof, door, rear, and two lateral walls of aluminum panels mounted to a white polypropylene base. The center panel of one of the walls had a speaker attached to a multiple tone generator and an Eiko 1820 miniature incandescent house light. A liquid dipper with a head entry detector was located centrally on the wall opposite of the speaker. Two retractable levers flanked the dipper; a triple LED stimulus light panel was positioned above each lever. A $0.01-\mathrm{ml}$ cup on the motorized arm of the liquid dipper provided reinforcement, which was a sweetened condensed milk (True Value ${ }^{\circledR}$ Walmart Brand, Bentonville, AR) and water mixture (one-third milk by volume). The operant chambers had a metal-wired floor and a stainless-steel waste pan filled with wood-chip bedding. The levers were set on continuous recording mode so that the duration of lever presses could be measured with a nominal resolution of $0.01 \mathrm{~s}$. A lever press was required to be separated by $0.06 \mathrm{~s}$ or more from the previous lever press in order to be counted as a new response. This threshold was selected because $0.06 \mathrm{~s}$ was the absolute minimum lever-press IRT previously observed in our laboratory (Brackney et al., 2011). The levers were calibrated to activate when a force of $0.2( \pm 0.05) \mathrm{N}$ was exerted on their edge.

\section{Procedure}

Throughout the experiment, lever presses were categorized as either super-threshold or subthreshold, where the threshold was $0.0,0.4$, or $0.8 \mathrm{~s}$, depending on the experimental condition (see Table 1). If the lever-press duration exceeded the threshold, the response was signaled by a flash of the lights above the lever and a $5-\mathrm{kHz}$ tone sounding for a brief but noticeable time $(0.1 \mathrm{~s})$. For the $0.0 \mathrm{~s}$ threshold, every lever press was immediately signaled.

Daily sessions began with a 5 -min acclimation period, during which no experimental events were programmed, followed by the extension of the left lever. Super-threshold lever pressing was reinforced on a VI 40-s schedule. Intervals were
Table 1 Training conditions

\begin{tabular}{lll}
\hline Duration threshold (s) & Cycle & Condition length (sessions) \\
\hline 0.0 & 1 & 15 \\
0.4 & 1 & 13 \\
0.8 & 1 & 13 \\
0.0 & 2 & 12 \\
0.4 & 2 & 12 \\
0.8 & 2 & 17 \\
\hline
\end{tabular}

Note. Experimental conditions occurred in descending order. When the duration threshold was $0.0 \mathrm{~s}$, a discrete response of any duration met the threshold requirement.

sampled without replacement from a 14-item list drawn from a Flesher-Hoffman distribution (Fleshler \& Hoffman, 1962). During reinforcement, the lever was withdrawn, the houselight illuminated and the dipper arm raised. Three seconds later, the dipper arm was lowered, the houselight turned off, and the lever reextended. Sessions terminated after $80 \mathrm{~min}$ or 84 reinforcer deliveries, whichever occurred first.

The response-duration threshold varied across six experimental conditions (see Table 1). During the first three conditions (Cycle 1) the duration threshold was 0.0, 0.4, and $0.8 \mathrm{~s}$. The following three conditions (Cycle 2) were replications of the previous three. Subjects were transitioned from one condition to the next after a minimum of 10 sessions, and when the mean response rate and the mean median response duration over the previous 5 days were judged stable by visual inspection.

\section{Data analysis}

All analyses were conducted on the responses of individual rats aggregated over the last five sessions of each condition. Estimates of bout-initiation rate $(b)$, within-bout response rate $(w)$, mean bout length $(L)$, and refractory period $(\delta)$ were based on the assumption that IRTs are distributed according to a shifted mixture of two exponential distributions (Eq. 2 in Brackney et al., 2011):

$$
\begin{aligned}
& \operatorname{Pr}(I R T=\tau \mid \tau<\delta)=0 \\
& \operatorname{Pr}(I R T=\tau \mid \tau \geq \delta)=p w e^{-w(\tau-\delta)}+(1-p) b e^{-b(\tau-\delta)} \\
& \min (\operatorname{IRT}) \geq \delta>0 ; w \geq b>0 ; 1 \geq p \geq 0 .
\end{aligned}
$$

where $L=p /(1-p)$ (see Appendix A in Cheung et al., 2012).

Equation 1 was fit to the distribution of IRTs using maximum likelihood estimation (Myung, 2003) with customwritten MATLAB ${ }^{\circledR}$ (MATLAB and Statistics Toolbox Release 2013, The MathWorks, Inc., Natick, MA) software. IRTs were defined as the intervals between the beginnings of each pair of consecutive super-threshold responses, excluding those with an intervening reinforcer; note that IRTs included 
response durations. ${ }^{1}$ Because estimates of $\delta$ were obtained from the shortest interval between super-threshold responses (see Fig. 1a), these estimates were expected to be very close to the 0.4-s and 0.8-s response-duration threshold in the corresponding conditions (see Table 1), and are not informative of sensorimotor capacity in those conditions. Nonetheless, $\delta$ was estimated and is reported as a manipulation check. Because $b$ and $w$ were estimated on the basis of the distribution of IRTs without $\delta$ (see Fig. 1a), these estimates were not expected to vary with response-threshold in a trivial manner.

Analyses of variance (ANOVAs) were conducted using Prism (GraphPad Software Inc., San Diego, CA). To identify significant effects, a 2 (cycle) $\times 3$ (threshold) repeatedmeasures ANOVA was conducted on each parameter estimate of interest. Simple main effects were assessed with Tukey's honestly significant difference test. Significant effects are reported when $p<.05$. ANOVAs were conducted on the logtransformed response rates, reinforcement rates, median response durations, interquartile range of response durations, and parameters estimates of Eq. 1, except $p$, which was logodds transformed. All variables of interest are reported backtransformed. A 2 (cycle) $\times 2$ (threshold) repeated-measures ANOVA was conducted on the arcsine-transformed ${ }^{2}$ proportion of responses that exceeded the response threshold for the 0.4 and $0.8 \mathrm{~s}$ threshold conditions (all responses were necessarily above the threshold in the $0.0 \mathrm{~s}$ threshold condition).

\section{Results}

\section{Response and reinforcement rates}

Overall response rate (computed including sub and superthreshold responses) and super-threshold response rate declined with longer duration thresholds; overall: $F(2,14)=$ 36.64, $p<.001$, super-threshold only: $F(2,14)=37.85, p<$ .001 (see Fig. 2a-b). Both dependent measures also declined between Cycles 1 and 2; overall: $F(1,7)=30.52, p<.001$, super-threshold only: $F(1,7)=28.53, p<.001$. Overall response rate declined significantly between the 0.0 and $0.8 \mathrm{~s}$ conditions and between the 0.4 and $0.8 \mathrm{~s}$ conditions in Cycle $1, p<.05$, and differed among all conditions in Cycle $2, p<$ .001 . Super-threshold response rates declined significantly with longer thresholds in both cycles, $p<.05$.

Reinforcement rate also declined with longer duration thresholds, $F(2,14)=13.43, p<.001$, and between cycles, $F(1,7)=9.80, p<.05$ (see Fig. 2c). Significant differences in reinforcement rate were observed between the 0.0 and $0.8 \mathrm{~s}$

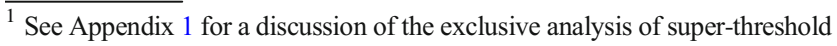
IRTs.

${ }_{2}^{2} y=\arcsin \left(x^{0.5}\right)$, where $x$ is a proportion and $y$ is approximately normally distributed.
}
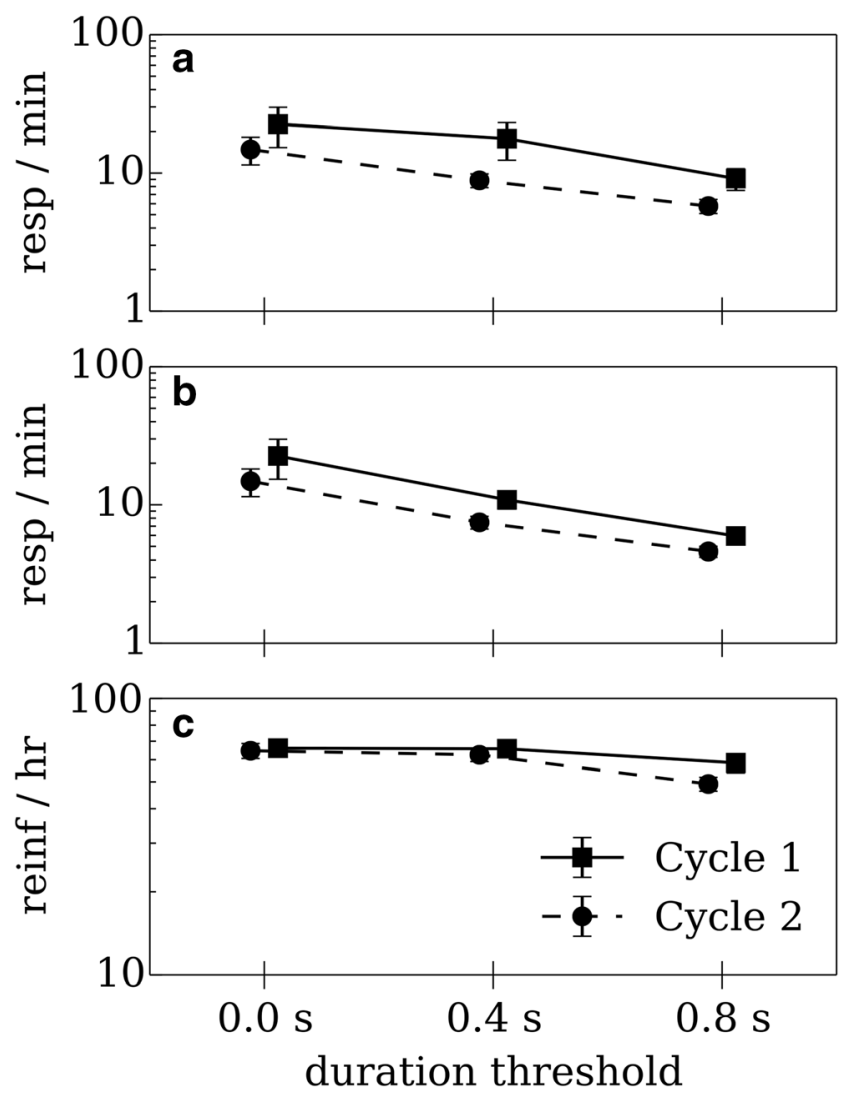

Fig. 2 Mean $( \pm$ SEM) median response and reinforcement rates as a function of response-duration threshold, calculated over the last five sessions in each threshold condition in Cycles 1 (solid curves) and 2 (dashed curves). a Overall response rate, calculated using both superthreshold and subthreshold responses. b Super-threshold response rate. c Reinforcement rate

conditions and between the 0.4 and $0.8 \mathrm{~s}, p<.05$. No significant Threshold $\times$ Cycle interaction effect was observed on either response rate or reinforcement rate.

\section{Response durations}

Figure 3 displays the distribution of response durations for the group and for two representative rats in each condition. In the $0.0 \mathrm{~s}$ conditions, the distribution of response durations appears to be approximately log-normal. Longer duration thresholds displaced a large portion of the distribution of response durations rightwards, just above the threshold. For the 0.4 and $0.8 \mathrm{~s}$ thresholds, response durations greater than the threshold appear to be log-normally distributed. Subthreshold response durations appear to be distributed according to a flatter distribution, which is distinct form the super-threshold durations.

Figure 4 displays summary statistics of the duration distributions: the mean $( \pm$ SEM) of the individual median durations (see Fig. 4a), the interquartile range of durations (Fig. 4b), and the proportion of durations that met or exceeded the duration threshold (Fig. 4c). Median response durations increased with longer duration thresholds: $F(2,14)=219.90, p<.001$. The 
group mean
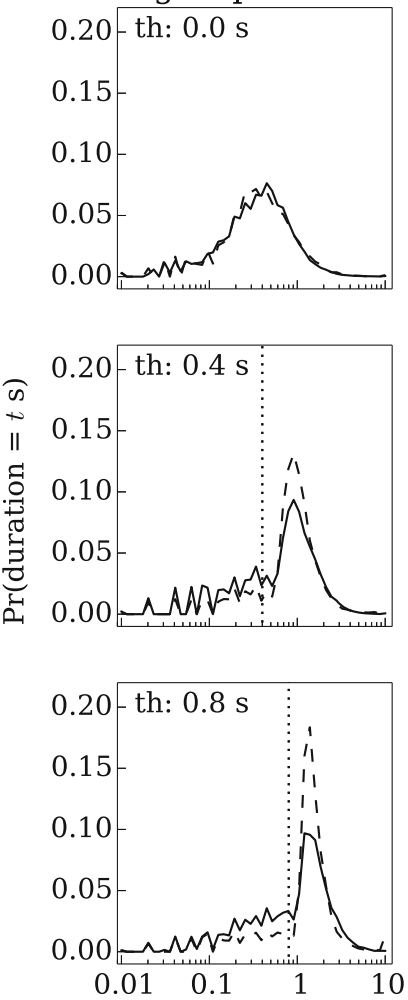

rat 2
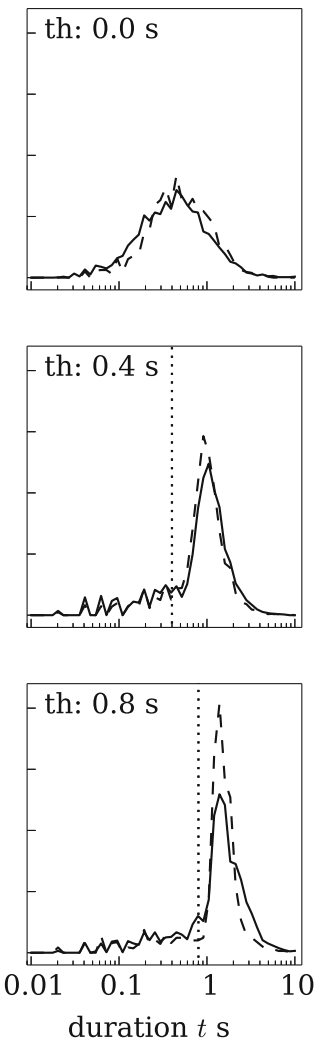

rat 7

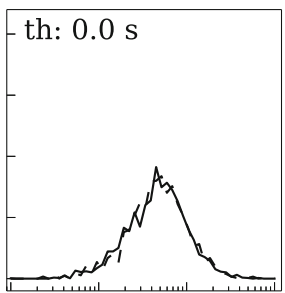

th: $0.4 \mathrm{~s}$
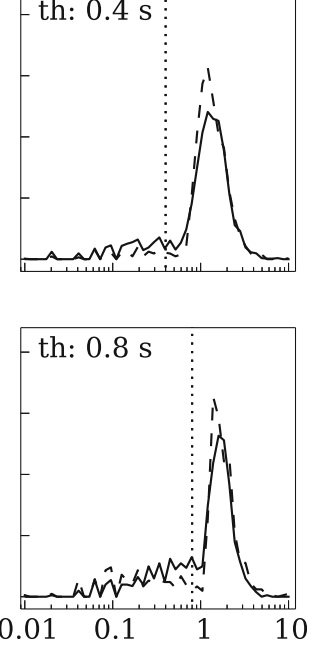

Fig. 3 Distribution of response durations in the last 5 days of each threshold condition in Cycles 1 (solid curves) and 2 (dashed curves). The vertical dotted lines indicate the response-duration threshold. The left column is the group means, the middle and right columns are representative rats. The abscissa is on a log scale to highlight the lognormal-like distribution of a portion of response durations interquartile range of the response durations also increased as the threshold increased, $F(2,14)=37.80, p<.001$, but declined between cycles, $F(1,7)=5.70, p<.05$. The proportion of responses that exceeded the duration threshold increased between cycles, $F(1,7)=29.49, p<.001$. These effects indicate that longer response-duration thresholds resulted in longer, more variable response durations, and point at potential learning effects between cycles.

\section{Response-bout modeling}

Figure 5 shows the mean $( \pm \mathrm{SEM})$ parameter estimates for each condition, based on fits of Eq. 1. Individual parameter estimates for each rat and condition are described in Tables 2 , 3 , and 4 of Appendix 2.

The average bout length, $L$, increased with longer duration thresholds, $F(2,14)=11.53, p<.05$. Significant differences in estimates of $L$ were observed between the 0.0 and $0.4 \mathrm{~s}$ conditions and between the 0.0 and $0.8 \mathrm{~s}$ conditions in both cycles, $p<.05$. Within-bout response rate, $w$, significantly decreased with longer thresholds, $F(2,14)=47.61, p<$ .001 , and between cycles; $F(1,7)=14.82, p<.05$. Significant differences in estimates of $w$ were observed among all conditions in Cycle $1, p<.05$, and between the 0.0 and
$0.4 \mathrm{~s}$ conditions and between the 0.0 and $0.8 \mathrm{~s}$ conditions in Cycle $2, p<.001$. Bout-initiation rate, $b$, significantly decreased with longer thresholds, $F(2,14)=34.12, p<.001$, and between cycles, $F(1,7)=14.89, p<.05$. Significant differences in estimates of $b$ were observed among all conditions in both cycles, $p<.05$. The refractory period, $\delta$, increased with longer duration thresholds, $F(2,14)=2599, p$ $<.001$, and between cycles, $F(1,7)=28.76, p<.001$. Significant differences in estimates of $\delta$ were observed among all threshold conditions in both cycles, $p<.001$. As expected, $\delta$ was close to the duration threshold when this was greater than zero. When considered together, the effects of responseduration threshold on $L, w$, and $b$ suggest that higher responseduration thresholds yielded fewer but longer response bouts that contained more spaced within-bout responses.

To ensure that the model was providing reasonable fits, log-survivor plots (Shull et al., 2001) of the model predictions were compared to log-survivor plots of the observed IRTs. Appendix 3 describes how model predictions were determined. Figure 6 displays the log survivor plots of the group mean and two representative rats for each duration threshold in Cycle 1. As the duration threshold increased, the shape of the log-survivor plot changed from the often-reported "broken-stick" pattern (e.g., Shull et al., 2001) to a straighter 

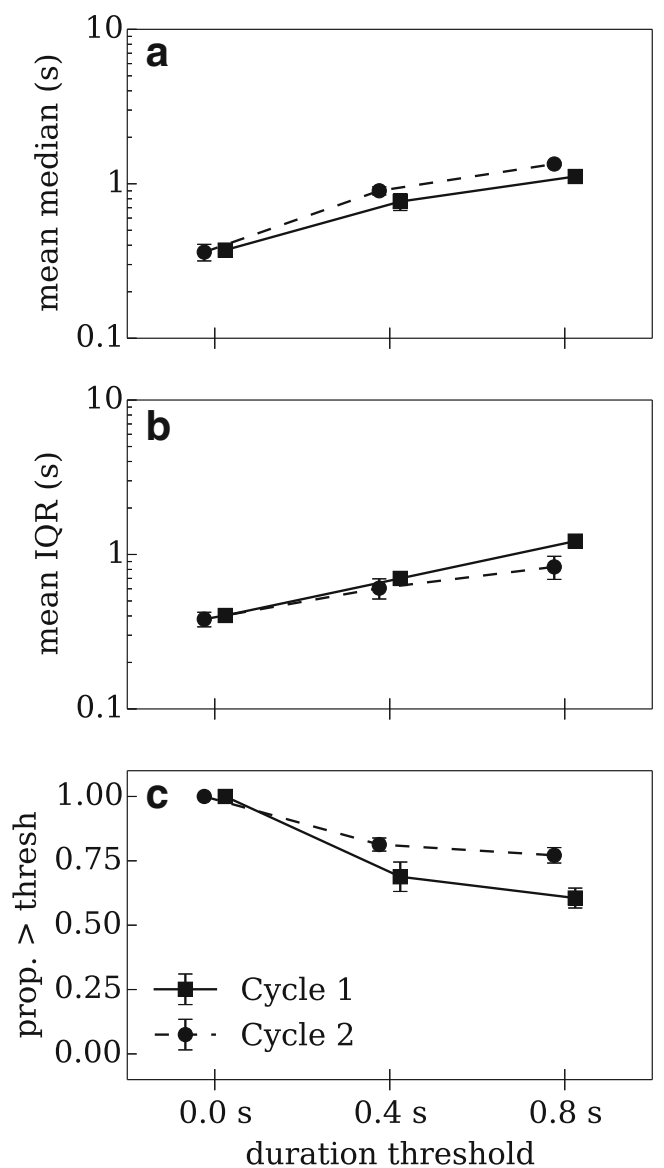

Fig. 4 Mean $( \pm$ SEM) of three response-duration distribution statistics as a function of response-duration threshold, calculated over the last five sessions in each threshold condition in Cycles 1 (solid curves) and 2 (dashed curves). a Median response duration; across all threshold conditions, the median duration was between 0.32 and $0.54 \mathrm{~s}$ longer than the required duration. $\mathbf{b}$ Interquartile range of response durations. $\mathbf{c}$ Proportion of responses that exceeded the duration threshold; all responses in the $0.0 \mathrm{~s}$ condition exceeded the threshold by design

pattern. The model faithfully reproduced the distribution of IRTs in the 0.4-s and 0.8-s conditions. In the 0.0-s condition, however, the simulation appears to overestimate the prevalence of the longest, approximately $20 \%$, of IRTs. Because the slope of the right-hand side of the "broken-stick" corresponds to $b$ (Shull et al., 2001), this divergence suggests that $b$ may be underestimated in the $0.0-\mathrm{s}$ condition. Nonetheless, regardless of condition, the shifted mixture of two exponential distributions (Eq. 1) was more likely to generate the observed IRTs than a single shifted exponential distribution,

$$
\begin{aligned}
& \operatorname{Pr}(\operatorname{IRT}=\tau \mid \tau<\delta)=0 \\
& \operatorname{Pr}(I R T=\tau \mid \tau \geq \delta)=\lambda e^{-\lambda(\tau-\delta)} . \\
& \quad \min (\operatorname{IRT}) \geq \delta>0 ; \delta>0 ; \lambda>0 .
\end{aligned}
$$

In Cycle 1, the likelihood ratio of Eq. 1 relative to Eq. 2 was 11,977 in the 0.0 -s condition, 4,189 in the 0.4 -s condition, and 1,269 in the 0.8 -s condition. Although the mixture- distribution model has 3 times more free parameters than the single-exponential model, based on the observed data, the former is at least $e^{1269}$ times more likely than the latter.

\section{Reanalyzing the log-survivor plot}

In log-survivor plots of IRT distributions, the vast majority of IRTs are represented in a small space in the upper left-hand portion of the plot. This feature of log-survivor plots helps emphasizes the "broken-stick" appearance that is characteristic of bi-exponentially distributed data (Shull et al., 2001), but exaggerates deviations from fit in IRTs corresponding to bout initiations, making it difficult to detect deviations from fit for within-bout IRTs. To identify the range of IRTs over which observation and model diverge, observed and modelpredicted IRTs were divided into bins each representing consecutive two percentile slices of the data; the mean IRT for each bin was calculated and plotted. This alternative method of comparing the observed and predicted IRTs allows the full range of IRTs to be more equally represented. The observed and predicted IRTs, organized in percentiles, are shown in Fig. 7 averaged across rats and for the two representative rats from Fig. 6. Figure 7 shows little deviation between data and model, suggesting that much of the apparent deviation in Fig. 6 is due to the "stretching out" of the longest IRTs.

\section{Discussion}

\section{Longer responses yield fewer but longer response bouts}

Increasing the operant lever-press duration requirement on a VI 40-s schedule of reinforcement lengthened the duration of lever presses (Fig. 4a) and, in consequence, also lengthened the shortest IRT (measured as the interval between the beginnings of consecutive responses that met the duration requirement; Fig. 5d). These effects simply show that rats were responsive to reinforcement contingencies. The question that motivated this study was whether response duration - the presumed expression of motoric manipulations in operant performance (Brackney et al., 2011; Posadas-Sanchez, 2004)affects other parameters of the microstructure of operant behavior, namely bout-initiation rate $(b)$, within-bout response rate $(w)$, and mean bout length $(L)$. Results show that all three parameters varied systematically with lever-press duration requirement, suggesting that longer responses are emitted at a lower rate in fewer but longer bouts (Fig. 5). Unlike changes in the shortest IRT ( $\delta$ ), changes in $b, w$, and $L$ are not implicit in the manipulation of duration requirement, because these parameters were estimated from the distribution of IRTs after subtracting $\delta$ (and, thus, the duration requirement; see Fig. 1). 

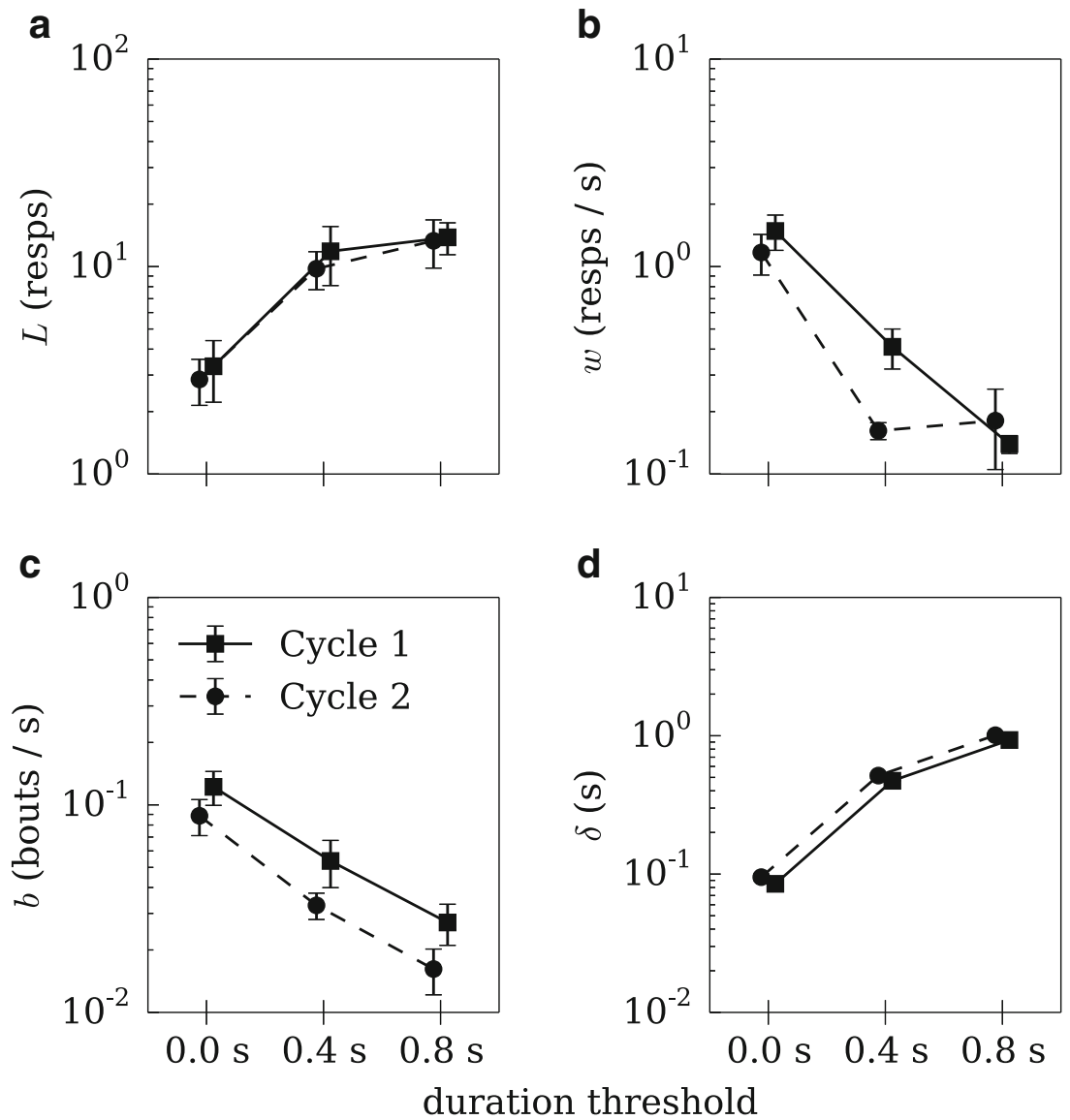

Fig. 5 Mean $( \pm$ SEM) parameter estimates (Eq. 1) as a function of response-duration threshold, computed from super-threshold IRTs in the last five days of each threshold condition in Cycles 1 (solid curves) and 2 (dashed curves). Bout length, not including the bout-initiation

These duration-requirement effects are only partially consistent with findings from three other comparable studies (Brackney et al., 2011; Jiménez et al., 2017; Pinkston \& Moore, 2020). Similar to long-duration requirements in the present study, increased motoric demands in previous studies yielded lower bout-initiation rates. However, Jiménez et al. (2017) observed shorter, not longer, response bouts with very high levers. It is important to note that lever-height effects were detected primarily at the maximum height tested in that study $-235 \mathrm{~mm}$, which was $20 \mathrm{~mm}$ above the mean maximum forepaw height of the Wistar rats that served as subjects. At lower heights (30-194 mm) no significant changes were observed in rate of reinforcement, response duration, $b, w$, or $L$, although certain effects on the distribution of bout length appear to emerge. Jiménez et al. (2017) speculated that there may be a critical lever height between 194 and $235 \mathrm{~mm}$ at which a motoric phase transition emerges, such that completion of the operant on higher levers do not simply take longer or involve more effort, but requires a qualitatively different response topography with broad impact on microstructural parameters. Below that critical height, the distribution of response durations and IRTs do not appear to vary significantly. Interestingly, the response (a), within-bout response rate (b), bout-initiation rate (c), and refractory period (d). Estimates for individual subjects are in Tables 2-4 of Appendix 2

relative height of concurrently available levers affects the microstructure of the operant behavior they support and the choice between them, even if they are below critical height (Jiménez, Ochoa, Amazeen, Amazeen, \& Cabrera, 2019).

Brackney et al. (2011) and Pinkston and Moore (2020) observed no significant effect of workload on bout length or within-bout response rates. The absence of significant effects of workload (lever height and force requirement) on $w$ and $L$ in Brackney et al. (2011) likely stems from the relatively small impact that their workload manipulation had on the refractory period. The higher workload condition in Brackney et al. (2011) did not increase the mean refractory period by more than $0.1 \mathrm{~s}$ in any experimental condition; the present study increased the mean refractory period by at least twice that difference. This may explain why even though Brackney et al. (2011) report a mean increase in bout length with higher workload, ${ }^{3}$ that increase was not statistically significant.

Pinkston and Moore (2020) manipulated the peak force required from responses to be reinforced (5.6 and $32.0 \mathrm{~g}$ ), but do not report response durations. It is possible that

\footnotetext{
${ }^{3}$ Estimated from parameter $q$, where $L=(1-q) / q$.
} 

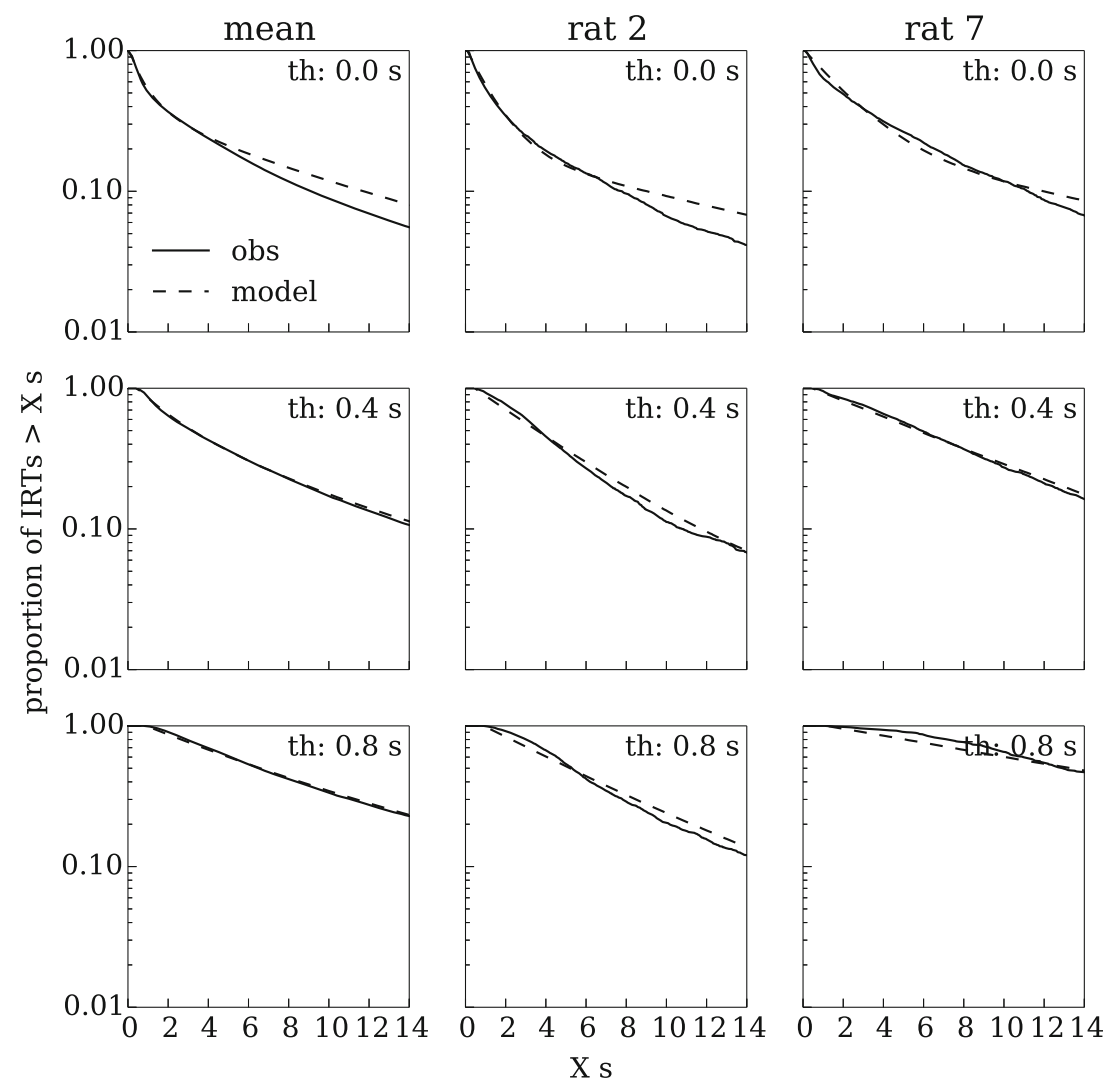

Fig. 6 Log survivor plots of super-threshold IRTs demonstrating model fit in Cycle 1. Solid curves correspond to empirically observed IRTs; dashed curves correspond to IRTs predicted by model simulation (see details in Appendix 3). Each row of plots corresponds to a different response-duration threshold. The left column is the group means, the

responses with peak forces within this range do not substantially vary in duration. In such case, their failure to observe force-requirement effects on bout length and within-bout response rate would be consistent with the notion that these parameters are more sensitive to the duration of the reinforced response than to the force required to complete it.

\section{Mechanisms underlying duration-threshold effects}

The most likely mechanism underlying the threshold-induced reduction in bout frequency appears to be somewhat intuitive. Brackney et al. (2011) suggested that reinforcer deprivation, availability, and response cost may drive what they labeled operant motivation, a general predisposition of the organism to engage the operandum, which is expressed in the boutinitiation rate. In the present study, higher duration thresholds may have raised the response cost, in the sense that longer responses entail a reduction in time that could be allocated to other activities.

Pinkston and Moore (2020) suggest an alternative explanation: The estimation of microstructural parameters based on criterial responses - those that are long or forceful enough to be reinforced-removes subcriterial responses from analysis, middle column and right columns are representative rats. Although some deviations from the observed data seem prominent from a visual inspection of the plots, they actually comprise only a small proportion of very long IRTs

lengthening IRTs even when behavior has not changed. It is thus possible that, when the duration threshold was raised in the present study, such IRT lengthening contributed to reducing the number of bouts of criterial responses. Nonetheless, when subcriterial responses were considered in their analysis, Pinkston and Moore (2020) also failed to find an effect of force requirement on overall response rate. In contrast, the present study shows that raising the duration requirement reduces overall response rate, even when subcriterial responses are counted (see Fig. 1a). Moreover, Pinkston and Moore's (2020) analysis is based on non-parametric statistical tests that may not be adequately powered to detect force-requirement effects on bout-initiation rate. A reanalysis of their data on VI 120 -s performance, implementing a one-tailed $t$ test on logtransformed estimates of $b$ from overall responses, yielded a significant $25 \%$ reduction in (back-transformed) estimates of $b$ with increased force requirement, $t(7)=2.15, p<.05$. Pinkston and Moore's (2020) data may not stand on its own as strong evidence for an effort-induced reduction in boutinitiation rate, but they are consistent with reports of such effect here and in Jiménez et al. (2017).

Potential mechanisms underlying the threshold-induced lengthening of bouts are perhaps less intuitive. Why would 

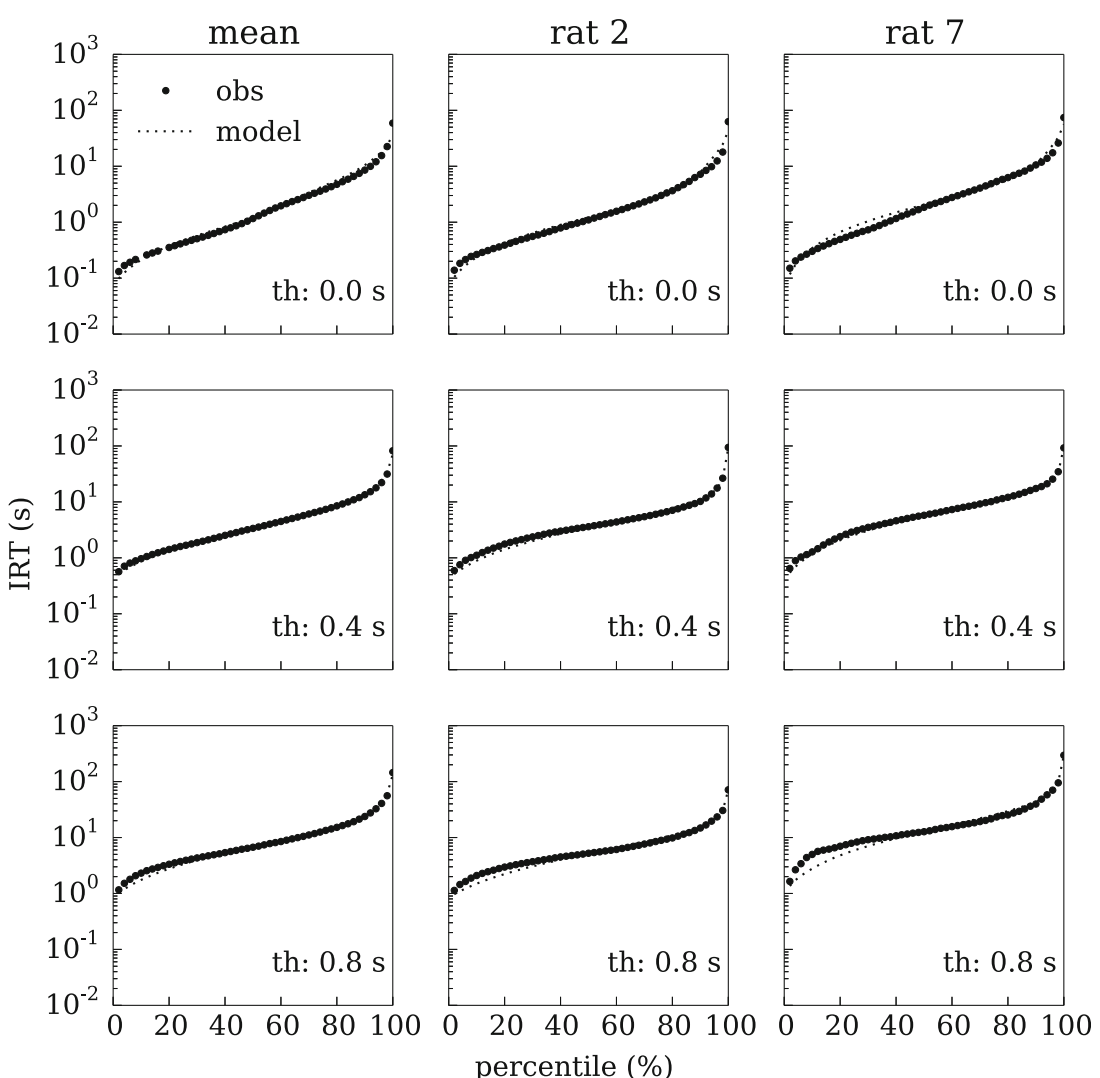

Fig. 7 Percentile plots of super-threshold IRTs demonstrating model fit in Cycle 1. Each point is the mean IRT for a two-percentile bin of the data. Heavy dots correspond to empirically observed IRTs; dotted curves correspond to IRTs predicted by model simulation. Each row of plots corresponds to a different response-duration threshold. The left column is the group means, the middle column and right columns are representative rats rats persist longer in a bout of more effortful and costly responses? One possibility involves the response-reinforcer coupling mechanism that Killeen posited as a mathematical principle of reinforcement (Killeen, 1994; Killeen \& Sitomer, 2003). This mechanism may be thought of as a limited memory buffer for recent responses; when a reinforcer is delivered, it strengthens all responses in the buffer according to their recency (for an alternative representation, see Catania, 2005). Longer responses presumably occupy a larger portion of the memory buffer and are thus more efficaciously reinforced; such efficacy may be reflected in longer bouts of those responses. This mechanism would also explain why bout length is proportional to the requirement of ratio schedules (Brackney \& Sanabria, 2015; Daniels \& Sanabria, 2017a; Reed, 2015; Shull et al., 2001), and why low-and presumably less effortful-ratio requirements yield lower response rates than intermediate ratio requirements (e.g., Sanabria, Acosta, Killeen, Neisewander, \& Bizo, 2008): Ratio schedules support short IRTs (Peele, Casey, \& Silberberg, 1984; Tanno, 2016), so responses fill up the memory buffer proportionally to the ratio requirement. Moreover, given the lower rate at which longer responses are emitted, more subthreshold responses may intervene between longer responses, potentially occupying the memory buffer, promoting reinforcement efficacy and longer bouts. That is, these subthreshold responses may effectively constitute a "self-imposed" variable-ratio requirement (Notterman \& Mintz, 1965; Pinkston \& Libman, 2017). To put it briefly, longer responses may be more memorable than shorter responses, so their reinforcement may yield longer bouts of responses.

It would be premature to speculate about the mechanism underlying the threshold-induced reduction of within-bout response, for two reasons. First, as the duration threshold increased, so did the proportion of subthreshold lever presses (Fig. 3), whose duration lengthened IRTs at higher thresholds. Such short lever presses are unlikely to contribute significantly to IRTs separating response bouts, but they are likely to substantially increase the IRTs separating responses within bouts. Second, Eq. 1 assumes that only the shortest IRT captures the time necessary to produce the reinforced response and to prepare the next response. Such assumption underestimates the typical duration and variability of reinforced responses (Figs. 3 and 4). Again, such underestimation is unlikely to contribute significantly to IRTs separating response bouts, but it may have affected the IRTs separating responses within bouts. It appears that, to better understand the 
mechanisms governing within-bout response rate, it is critical to develop an account of subthreshold behavior and response duration. Such development will probably require the direct manipulation of motoric requirements, which involves substantial challenges for the measurement, analysis, and interpretation of behavioral changes. A particularly important challenge is that those requirements define the operant response (Pinkston \& Foss, 2018; Pinkston \& Libman, 2017); manipulating motoric requirements entails changing what is considered an IRT, which is the basis of the microstructural model implemented here.

\section{Responses have variable durations}

In regard to the provenance of subthreshold behavior, training order cannot be discounted as a potential contributor. Although behavior adjusted to changes in duration threshold, shorter responses reinforced in earlier experimental conditions with lower duration thresholds (see Table 1) may have persisted into later conditions within the same cycle. Subthreshold responses may have been induced through the same memory-buffer mechanism responsible for thresholdinduced bout length: the reinforcement of super-threshold responses may have strengthened the subthreshold responses that preceded it. Gharib and colleagues' findings suggest yet another source of variability in response durations (Gharib, Derby, \& Roberts, 2001; Gharib, Gade, \& Roberts, 2004; Roberts \& Gharib, 2006). Roberts and Gharib (2006) hypothesized that decreasing the probability of reinforcement increases the variability in response durations. The rate of earned reinforcers (Fig. 2c) decreased significantly (if by a small margin) as the response threshold increased, which may have increased the variability of response durations.

Regardless of its cause, the variability in response durations is inconsistent with the assumption of Eq. 1 that, under constant conditions, the refractory period is constant. Instead, it appears that response duration is a mixture-distributed random variable with at least two components: a shifted log-normal distribution of super-threshold latencies, and an unknown but flatter distribution of durations that is insensitive to threshold requirement. Future research may determine whether the parameters of this mixture distribution are sensitive to motivational and schedule manipulations, as suggested previously (Faustman \& Fowler, 1981; Roberts \& Gharib, 2006).

\section{Conclusion}

Reinforced responses are organized in bouts. The selective reinforcement of longer response durations not only increases the relative frequency of these durations, it also yields fewer but longer response bouts. Bout-length effects may reflect a duration-induced facilitation of the response-outcome association. It is possible that, within certain limits, effortful responses lengthen response bouts through a similar mechanism: If those responses take longer to complete, they may be more likely to be associated with reinforcement.

The variability in response durations indicates that, contrary to prior assumptions, the refractory period between IRTs is not constant. Previous studies have shown that response durations can provide valuable information about the behavioral processes involved in operant performance (e.g., Roberts \& Gharib, 2006). Future research may explore how the variability of the refractory period is integrated into more comprehensive models of the microstructure of reinforced behavior.

\section{Appendix 1 Selection of super-threshold IRTs for analysis}

Model fits were conducted only on the IRTs between superthreshold responses to more accurately reflect operant performance as it is typically recorded. In most operant protocols, it is likely that animals regularly produce behaviors that belong to the target response class, but go unnoticed by the experimenter. When a discrete switch closure constitutes the functional response that triggers reinforcement, there may be a multitude of behaviors that are either induced due to their temporal proximity to the response that triggered reinforcement (Catania, 1971; Killeen \& Pellón, 2013) or due to generalizations of the functional response (e.g., responses that are topographically similar to the target, but do not meet the reinforcement criterion). When lever tension is tightened to increase the force needed to depress the lever, as in Brackney et al. (2011), the subject may continue to emit responses that no longer fully depress the lever and hence go unrecorded. Pinkston and colleagues, among others, have demonstrated this methodological challenge by programming reinforcement contingent upon exerting a certain force on a force transducer (Pinkston \& Foss, 2018; Pinkston \& Libman, 2017; Pinkston $\&$ Moore, 2020). In these studies, a significant proportion of responses was below the force threshold, and would not have been identified with a more typical operandum. For these reasons, functionally ineffective subthreshold responses were excluded from response-bout modeling.

\section{Appendix 2 Parameter estimates for individual subjects}

Estimates of individual parameters of Eq. 1, for each threshold condition and cycle. Estimates were obtained using the method of maximum likelihood. 
Table 2 Threshold $=0.0 \mathrm{~s}$

\begin{tabular}{|c|c|c|c|c|c|c|c|c|}
\hline \multirow[t]{2}{*}{ Rat } & \multicolumn{4}{|c|}{ Cycle 1} & \multicolumn{4}{|c|}{ Cycle 2} \\
\hline & $L$ & $w$ & $b$ & $\delta$ & $L$ & $w$ & $b$ & $\delta$ \\
\hline 1 & 10.65 & 2.23 & 0.27 & 0.08 & 3.21 & 2.10 & 0.17 & 0.0 \\
\hline 2 & 4.11 & 0.79 & 0.08 & 0.09 & 1.03 & 0.86 & 0.08 & 0.11 \\
\hline 3 & 0.88 & 1.75 & 0.14 & 0.08 & 1.35 & 2.25 & 0.08 & 0.09 \\
\hline 4 & 3.31 & 0.87 & 0.10 & 0.09 & 2.23 & 1.19 & 0.14 & 0.10 \\
\hline 5 & 1.14 & 1.27 & 0.08 & 0.08 & 3.32 & 0.30 & 0.03 & 0.12 \\
\hline 6 & 0.85 & 3.11 & 0.09 & 0.09 & 1.94 & 0.75 & 0.06 & 0.08 \\
\hline 7 & 4.18 & 0.44 & 0.06 & 0.09 & 7.82 & 0.19 & 0.03 & 0.10 \\
\hline 8 & 1.34 & 1.40 & 0.17 & 0.08 & 1.95 & 1.71 & 0.13 & 0.08 \\
\hline Mean & 3.31 & 1.48 & 0.12 & 0.08 & 2.86 & 1.17 & 0.09 & 0.09 \\
\hline Median & 2.33 & 1.34 & 0.10 & 0.08 & 2.09 & 1.03 & 0.08 & 0.09 \\
\hline SEM & 1.16 & 0.31 & 0.02 & $<0.00$ & 0.76 & 0.28 & 0.02 & 0.01 \\
\hline
\end{tabular}

Note. The units for $L, w, b$, and $\delta$ are responses, responses/s, bouts/s, and $\mathrm{s}$, respectively.

Table 3 Threshold $=0.4 \mathrm{~s}$

\begin{tabular}{|c|c|c|c|c|c|c|c|c|}
\hline \multirow[t]{2}{*}{ Rat } & \multicolumn{4}{|c|}{ Cycle 1} & \multicolumn{4}{|c|}{ Cycle 2} \\
\hline & $L$ & $w$ & $b$ & $\delta$ & $L$ & $w$ & $b$ & $\delta$ \\
\hline 1 & 3.33 & 0.82 & 0.14 & 0.47 & 18.80 & 0.15 & 0.04 & 0.48 \\
\hline 2 & 22.94 & 0.24 & 0.02 & 0.47 & 9.69 & 0.18 & 0.03 & 0.51 \\
\hline 3 & 0.70 & 0.76 & 0.09 & 0.47 & 2.17 & 0.18 & 0.04 & 0.49 \\
\hline 4 & 10.19 & 0.48 & 0.05 & 0.47 & 12.51 & 0.25 & 0.05 & 0.47 \\
\hline 5 & 7.59 & 0.18 & 0.02 & 0.49 & 7.52 & 0.15 & 0.01 & 0.47 \\
\hline 6 & 3.27 & 0.48 & 0.05 & 0.47 & 5.99 & 0.15 & 0.02 & 0.48 \\
\hline 7 & 33.85 & 0.14 & 0.02 & 0.47 & 17.60 & 0.09 & 0.01 & 0.69 \\
\hline 8 & 12.88 & 0.19 & 0.05 & 0.47 & 3.81 & 0.14 & 0.05 & 0.53 \\
\hline Mean & 11.84 & 0.41 & 0.05 & 0.47 & 9.76 & 0.16 & 0.03 & 0.51 \\
\hline Median & 8.89 & 0.36 & 0.05 & 0.47 & 8.61 & 0.15 & 0.03 & 0.49 \\
\hline SEM & 4.01 & 0.10 & 0.01 & $<0.00$ & 2.17 & 0.02 & 0.01 & 0.03 \\
\hline
\end{tabular}

Note. The units for $L, w, b$, and $\delta$ are responses, responses/s, bouts/s, and $\mathrm{s}$, respectively.
Table $4 \quad$ Threshold $=0.8 \mathrm{~s}$

\begin{tabular}{|c|c|c|c|c|c|c|c|c|}
\hline \multirow[t]{2}{*}{ Rat } & \multicolumn{4}{|c|}{ Cycle 1} & \multicolumn{4}{|c|}{ Cycle 2} \\
\hline & $L$ & $w$ & $b$ & $\delta$ & $L$ & $w$ & $b$ & $\delta$ \\
\hline 1 & 21.30 & 0.17 & 0.04 & 0.87 & 33.83 & 0.13 & 0.01 & 0 . \\
\hline 2 & 20.11 & 0.17 & 0.03 & 0.91 & 13.03 & 0.10 & 0.01 & 0.9 \\
\hline 3 & 10.90 & 0.13 & 0.01 & 0.88 & 21.71 & 0.08 & 0.01 & 1.0 \\
\hline 4 & 18.89 & 0.19 & 0.03 & 0.93 & 11.63 & 0.13 & 0.01 & 1.1 \\
\hline 5 & 6.60 & 0.15 & 0.02 & 0.87 & 0.40 & 0.74 & 0.04 & 0.8 \\
\hline 6 & 4.38 & 0.10 & 0.02 & 0.89 & 5.52 & 0.10 & 0.02 & 1.1 \\
\hline 7 & 21.42 & 0.06 & 0.01 & 1.17 & 14.22 & 0.07 & 0.00 & 1.12 \\
\hline 8 & 7.01 & 0.15 & 0.06 & 0.93 & 6.12 & 0.08 & 0.02 & 0.95 \\
\hline Mean & 13.83 & 0.14 & 0.03 & 0.93 & 13.31 & 0.18 & 0.02 & 1.0 \\
\hline Median & 14.90 & 0.15 & 0.03 & 0.90 & 12.33 & 0.10 & 0.01 & 0.9 \\
\hline SEM & 2.59 & 0.01 & 0.01 & 0.04 & 3.72 & 0.08 & 0.00 & 0.0 \\
\hline
\end{tabular}

Note. The units for $L, w, b$, and $\delta$ are responses, responses/s, bouts/s, and $\mathrm{s}$, respectively.

\section{Appendix 3 IRT simulations}

Simulations were conducted to obtain the predicted distribution of IRTs from the fitted model (Eq. 1). Although determining the expected inverse cumulative distribution (i.e., a survivor plot) is a simple matter of integrating over the probability density function, determining the mean IRT for each percentile bin is more complicated. To keep estimation techniques identical for both the survival plots (Fig. 6) and percentile plots (Fig. 7), IRT-generation simulations were used for both types of plots.

Each simulation used a Monte Carlo method in which a series of Bernoulli trials were generated. After each success [with probability $p=L /(L+1)$ ], an IRT was sampled from an exponential distribution with a mean of $1 / w$; after each failure (with probability $1-p$ ) an IRT was sampled from an exponential distribution with a mean of $1 / b$. The refractory period, $\delta$, was then added to the IRT. The sampled IRT then advanced the session clock by its respective value, and a new trial then began. The session continued until the session clock exceeded the maximum session length of $3,160 \mathrm{~s}$ (the 84-min session time minus the 5-min acclimation period); the vector of IRTs was truncated to exclude the final IRT, which advanced the session clock beyond the session length. One hundred sessions were simulated for each rat and condition; the generated IRTs were then aggregated to produce the model prediction traces for the log survivor and percentile plots in Figs. 6 and 7.

Author note This study was supported by two grants from the National Institutes of Health to Federico Sanabria (DA032632, MH094562). We would like to thank Peter Killeen and Janet Neisewander for their helpful 
comments and suggestions. We would like to thank Alexander Gilmour, Alexander Spitzer, Alexandra Paul, Christopher Fencl, Fritzgerald Jerome, Gabriel Wood-Isenberg, Marie Simonsen, Gabriel Mazur, and Elizabeth Watterson for their assistance in data collection.

Open practices statement All data are available upon request to the corresponding author.

\section{References}

Brackney, R. J., Cheung, T. H. C., Neisewander, J. L., \& Sanabria, F. (2011). The isolation of motivational, motoric, and schedule effects on operant performance: A modeling approach. Journal of the Experimental Analysis of Behavior, 96(1), 17-38. https://doi.org/ 10.1901/jeab.2011.96-17

Brackney, R. J., Cheung, T. H. C., \& Sanabria, F. (2017). A bout analysis of operant response disruption. Behavioural Processes, 141(Part 1): 42-49. https://doi.org/10.1016/j.beproc.2017.04.008

Brackney, R. J., \& Sanabria, F. (2015). The distribution of response bout lengths and its sensitivity to differential reinforcement. Journal of the Experimental Analysis of Behavior, 104(2), 167-185. https://doi. org/10.1002/jeab.168

Byrne, T., \& Sarno, B. (2019). Response duration is sensitive to both immediate and delayed reinforcement. Journal of the Experimental Analysis of Behavior, 111(1), 94-115. https://doi.org/10.1002/jeab. 491

Catania, A. C. (1971). Reinforcement schedules: The role of responses preceding the one that produces the reinforcer. Journal of the Experimental Analysis of Behavior, 15(3), 271-287. https://doi. org/10.1901/jeab.1971.15-271

Catania, A. C. (2005). The operant reserve: A computer simulation in (accelerated) real time. Behavioural Processes, 69(2), 257-278. https://doi.org/10.1016/j.beproc.2005.02.009

Chen, X., \& Reed, P. (2020). Factors controlling the micro-structure of human free-operant behaviour: Bout-initiation and within-bout responses are effected by different aspects of the schedule. Behavioural Processes, 175, 104106. https://doi.org/10.1016/j. beproc. 2020.104106

Cheung, T. H. C., Neisewander, J. L., \& Sanabria, F. (2012). Extinction under a behavioral microscope: Isolating the sources of decline in operant response rate. Behavioural Processes, 90(1), 111-123. https://doi.org/10.1016/j.beproc.2012.02.012

Daniels, C. W., \& Sanabria, F. (2017a). About bouts: A heterogeneous tandem schedule of reinforcement reveals dissociable components of operant behavior in Fischer rats. Journal of Experimental Psychology: Animal Learning and Cognition, 43(3), 280-294. https://doi.org/10.1037/xan0000144

Daniels, C. W., \& Sanabria, F. (2017b). Interval timing under a behavioral microscope: Dissociating motivational and timing processes in fixed-interval performance. Learning \& Behavior, 45(1), 29-48. https://doi.org/10.3758/s13420-016-0234-1

Faustman, W. O., \& Fowler, S. C. (1981). Use of operant response duration to distinguish the effects of haloperidol from nonreward. Pharmacology Biochemistry and Behavior, 15(2), 327-329. https://doi.org/10.1016/0091-3057(81)90196-9

Fleshler, M., \& Hoffman, H. S. (1962). A progression for generating variable-interval schedules. Journal of the Experimental Analysis of Behavior, 5(4), 529-530. https://doi.org/10.1901/jeab.1962.5529

Fowler, S. C., Filewich, R. J., \& Leberer, M. R. (1977). Drug effects upon force and duration of response during fixed-ratio performance in rats. Pharmacology Biochemistry and Behavior, 6(4), 421-426. https://doi.org/10.1016/0091-3057(77)90179-4

Gharib, A., Derby, S., \& Roberts, S. (2001). Timing and the control of variation. Journal of Experimental Psychology: Animal Behavior Processes, 27(2), 165-178. Article isi:000170959500006

Gharib, A., Gade, C., \& Roberts, S. (2004). Control of variation by reward probability. Journal of Experimental Psychology: Animal Behavior Processes, 30(4), 271-282. https://doi.org/10.1037/00977403.30.4.271 Retrieved from isi:000170959500006

Herrnstein, R. J. (1970). On the law of effect. Journal of the Experimental Analysis of Behavior, 13(2), 243-266. https://doi.org/10.1901/jeab. 1970.13-243

Hoffman, D. J., \& Newland, M. C. (2016). A microstructural analysis distinguishes motor and motivational influences over voluntary running in animals chronically exposed to methylmercury and nimodipine. NeuroToxicology, 54, 127-139. https://doi.org/10. 1016/j.neuro.2016.04.009

Íbias, J., Daniels, C. W., Miguéns, M., Pellón, R., \& Sanabria, F. (2017). The effect of methylphenidate on the microstructure of scheduleinduced polydipsia in an animal model of ADHD. Behavioural Brain Research, 333, 211-217. https://doi.org/10.1016/j.bbr.2017. 06.048

Jiménez, Á. A., Sanabria, F., \& Cabrera, F. (2017). The effect of lever height on the microstructure of operant behavior. Behavioural Processes, 140, 181-189. https://doi.org/10.1016/j.beproc.2017. 05.002

Jiménez, A. A., Ochoa, D. A., Amazeen, P. G., Amazeen, E. L, \& Cabrera, F. (2019). Affordances guide choice behavior between equal schedules of reinforcement in rats. Ecological Psychology, 31(4), 316-331. https://doi.org/10.1080/10407413.2019.1599686.

Johnson, J. E., Pesek, E. F., \& Christopher Newland, M. (2009). Highrate operant behavior in two mouse strains: A response-bout analysis. Behavioural Processes, 81(2), 309-315. https://doi.org/10. 1016/j.beproc.2009.02.013

Killeen, P. R. (1994). Mathematical principles of reinforcement: Based on the correlation of behavior with incentives in short-term memory. Behavioral and Brain Sciences, 17(1), 105-172. https://doi.org/10. 1017/S0140525X00033628

Killeen, P. R., Hall, S. S., Reilly, M. P., \& Kettle, L. C. (2002). Molecular analyses of the principal components of response strength. Journal of the Experimental Analysis of Behavior, 78(2), 127-160. https:// doi.org/10.1901/jeab.2002.78-127

Killeen, P. R., \& Pellón, R. (2013). Adjunctive behaviors are operants. Learning \& Behavior, 41(1), 1-24. https://doi.org/10.3758/s13420012-0095-1

Killeen, P. R., \& Sitomer, M. T. (2003). MPR. Behavioural Processes, 62(1/3), 49-64. https://doi.org/10.1016/S0376-6357(03)00017-2

Matsui, H., Yamada, K., Sakagami, T., \& Tanno, T. (2018). Modeling bout-pause response patterns in variable-ratio and variable-interval schedules using hierarchical Bayesian methodology. Behavioural Processes, 157, 346-353. https://doi.org/10.1016/j.beproc.2018. 07.014

Myung, I. J. (2003). Tutorial on maximum likelihood estimation. Journal of Mathematical Psychology, 47(1), 90-100. https://doi.org/10. 1016/S0022-2496(02)00028-7

Notterman, J. M., \& Mintz, D. E. (1965). Dynamics of response. New York, NY: Wiley.

Peele, D. B., Casey, J., \& Silberberg, A. (1984). Primacy of interresponse-time reinforcement in accounting for rate differences under variable-ratio and variable-interval schedules. Journal of Experimental Psychology: Animal Behavior Processes, 10(2), 149-167. https://doi.org/10.1037/0097-7403.10.2.149

Pinkston, J. W., \& Foss, E. K. (2018). The role of response force on the persistence and structure of behavior during extinction. Journal of the Experimental Analysis of Behavior, 109(1), 194-209. https://doi. org/10.1002/jeab.306 
Pinkston, J. W., \& Libman, B. M. (2017). Aversive functions of response effort: Fact or artifact? Journal of the Experimental Analysis of Behavior, 108(1), 73-96. https://doi.org/10.1002/jeab.264

Pinkston, J. W., \& Moore, A. (2020). The effects of force requirements on microstructure: Subcriterion responses and the mismeasurement of bouts. Journal of the Experimental Analysis of Behavior, 114(1), 323. https://doi.org/10.1002/jeab.613

Posadas-Sanchez, D. (2004). Evaluating models of motivation: Role of reinforcer quality, lever force and drug effects. Tempe, AZ: Arizona State University.

Reed, P. (2011). An experimental analysis of steady-state response rate components on variable ratio and variable interval schedules of reinforcement. Journal of Experimental Psychology: Animal Behavior Processes, 37(1), 1-9. https://doi.org/10.1037/a0019387

Reed, P. (2015). The structure of random ratio responding in humans. Journal of Experimental Psychology: Animal Learning and Cognition, 41(4), 419-431. https://doi.org/10.1037/xan0000081

Reed, P., Smale, D., Owens, D., \& Freegard, G. (2018). Human performance on random interval schedules. Journal of Experimental Psychology: Animal Learning and Cognition, 44(3), 309-321. https://doi.org/10.1037/xan0000172

Roberts, S., \& Gharib, A. (2006). Variation of bar-press duration: Where do new responses come from? Behavioural Processes, 72(3), 215223. https://doi.org/10.1016/j.beproc.2006.03.003

Romero, K., Daniels, C. W., Gipson, C. D., \& Sanabria, F. (2018). Suppressive and enhancing effects of nicotine on food-seeking behavior. Behavioural Brain Research, 339, 130-139. https://doi.org/ 10.1016/j.bbr.2017.11.023

Sanabria, F. (2019). Primer on learning and conditioning: A quantitative approach. San Diego, CA: Cognella.

Sanabria, F., Acosta, J. I. J. I., Killeen, P. R. P. R., Neisewander, J. L. J. L., \& Bizo, L. A. L. A. (2008). Modeling the effects of fluoxetine on food-reinforced behavior. Behavioural Pharmacology, 19(1), 6170. https://doi.org/10.1097/FBP.0b013e3282f3df9b

Sanabria, F., Daniels, C. W., Gupta, T., \& Santos, C. (2019). A computational formulation of the behavior systems account of the temporal organization of motivated behavior. Behavioural Processes, 169, 103952. https://doi.org/10.1016/j.beproc.2019.103952

Shull, R. L. (2004). Bouts of responding on variable-interval schedules: Effects of deprivation level. Journal of the Experimental Analysis of Behavior, 81(2), 155-167. https://doi.org/10.1901/jeab.2004.81155

Shull, R. L., Gaynor, S. T., \& Grimes, J. A. (2001). Response rate viewed as engagement bouts: Effects of relative reinforcement and schedule type. Journal of the Experimental Analysis of Behavior, 75(3), 247274. https://doi.org/10.1901/jeab.2001.75-247

Shull, R. L., \& Grimes, J. A. (2003). Bouts of responding from variableinterval reinforcement of lever pressing by rats. Journal of the Experimental Analysis of Behavior, 80(2), 159-171. https://doi. org/10.1901/jeab.2003.80-159

Shull, R. L., Grimes, J. A., \& Bennett, J. A. (2004). Bouts of responding: The relation between bout rate and the rate of variable-interval reinforcement. Journal of the Experimental Analysis of Behavior, 81(1), 65-83. https://doi.org/10.1901/jeab.2004.81-65

Smith, T. T., McLean, A. P., Shull, R. L., Hughes, C. E., \& Pitts, R. C. (2014). Concurrent performance as bouts of behavior. Journal of the Experimental Analysis of Behavior, 102(1), 102-125. https://doi. org $/ 10.1002 /$ jeab. 90

Tanno, T. (2016). Response-bout analysis of interresponse times in variable-ratio and variable-interval schedules. Behavioural Processes, 132, 12-21.

Publisher's note Springer Nature remains neutral with regard to jurisdictional claims in published maps and institutional affiliations. 